\title{
Next-to-leading order slepton pair production at hadron colliders
}

\author{
Howard Baer and B. W. Harris \\ Department of Physics, Florida State University, Tallahassee, Florida 32306-4350 \\ Mary Hall Reno \\ Department of Physics and Astronomy, University of Iowa, Iowa City, Iowa 52242 \\ and Theory Division, CERN, CH-1211 Geneva 23, Switzerland
}

(Received 9 December 1997; published 27 March 1998)

\begin{abstract}
We compute total cross sections for various slepton pair production reactions $\widetilde{\ell}_{L} \widetilde{\nu}_{L}, \widetilde{\ell}_{L} \overline{\mathscr{\ell}}_{L}, \widetilde{\ell}_{R} \overline{\mathscr{\ell}}_{R}$ and $\widetilde{\nu}_{L} \overline{\tilde{\nu}}_{L}$ in next-to-leading order QCD. For $p \bar{p}$ collisions at $\sqrt{S}=2 \mathrm{TeV}$, we find leading order cross sections to be enhanced by typically $35 \%$ to $40 \%$. For $p p$ collisions at $\sqrt{S}=14 \mathrm{TeV}$, the enhancement ranges from $25 \%$ to $35 \%$ depending on the mass of the sleptons. We comment upon the phenomenological implications of these results. [S0556-2821(98)02311-X]
\end{abstract}

PACS number(s): 14.80.Ly, 12.38.Bx, 13.85.Qk

\section{INTRODUCTION}

Particle physics models that incorporate weak scale supersymmetry (SUSY) are certainly among the most compelling possibilities for physics beyond the standard model (SM) [1]. Numerous searches for the sparticles of supersymmetric models have been made at both lepton and hadron colliders. The lack of any convincing supersymmetric signal beyond standard model backgrounds has led to a variety of limits on sparticle masses [2].

The best search limits on strongly interacting superpartners, the squarks $\widetilde{q}$ and gluinos $\widetilde{g}$, have been obtained from the Collider Detector at Fermilab (CDF) and D0 experiments at the Fermilab Tevatron $p \bar{p}$ collider. By searching for multijet $+\boldsymbol{E}_{T}$ events, limits of $m_{\tilde{g}} \geqslant 144-154 \mathrm{GeV}$ (for $m_{\tilde{q}}$ $\gg m_{g}$ ) and $m_{\tilde{q}}>212-224 \mathrm{GeV}$ (for $m_{\tilde{q}} \simeq m_{\tilde{g}}$ ) have been found $[3,4]$. Recently, a next-to-leading order computation of $\widetilde{g} \widetilde{g}, \widetilde{g} \widetilde{q}$ and $\widetilde{q} \widetilde{q}$ production has been completed [5]. As a result of this calculation, the theoretical uncertainty due to choice of renormalization and factorization scales has been reduced. In addition, for a scale choice of the order of the average mass of the produced sparticles, the pair production cross sections were found to increase by a factor of 1 to 2 depending upon the subprocesses and collider. This has led to $10-30 \mathrm{GeV}$ improvements in the mass limits coming from the CDF and D0 collaborations.

For weakly interacting sparticles such as the charginos $\widetilde{W}_{1}$ and sleptons $\widetilde{\ell}_{R}, \widetilde{\ell}_{L}$ and $\widetilde{\nu}_{\ell L}(\ell=e, \mu$ or $\tau)$, the best search limits come from experiments at the CERN $e^{+} e^{-}$ collider (LEP2). Currently, $m_{\widetilde{W}_{1}}>85.5 \mathrm{GeV}$ for a gauginolike chargino [6]. For charged sleptons, $m_{\tilde{e}_{R}} \gtrsim 75 \mathrm{GeV}$, $m_{\tilde{\mu}_{R}} \gtrsim 59 \mathrm{GeV}$ and $m_{\tilde{\tau}_{R}} \gtrsim 53 \mathrm{GeV}$ (where the exact bounds depend on the lightest neutralino mass $m_{\widetilde{Z}_{1}}$ ) [7]. In addition, sneutrinos are required to have mass $m_{\nu} \gtrsim 41.8 \mathrm{GeV}$ from measurements of the invisible $Z$ width [8] (three degenerate generations of invisibly decaying sneutrinos have been assumed). Search limits from the Tevatron collider on charginos and neutralinos can be obtained by looking for clean trilepton events from $\widetilde{W}_{1} \widetilde{Z}_{2} \rightarrow 3 \ell+\mathbb{E}_{T}$ production. Expected event rates are very model dependent, so that limits are best placed as contours in parameter space of specific models [9].

The possibility of searching for slepton production at hadron colliders has been studied in Refs. [10,11]. In gravitymediated models of supersymmetry breaking (SUGRA models) at Tevatron conditions, direct slepton pair production occurs at low rates, although it may be possible to extract a signal at one of the upgrade options such as the main injector, which will operate at $\sqrt{S}=2 \mathrm{TeV}$ and accumulate $\sim 2$ $\mathrm{fb}^{-1}$ of integrated luminosity, or TeV33, which will operate at the same energy, but amass $\sim 25 \mathrm{fb}^{-1}$ of integrated luminosity [11]. For models where SUSY breaking is communicated by gauge interactions [12], slepton pair production can lead to final states with two isolated photons plus two isolated leptons, a signature for which SM backgrounds are expected to be small [13].

For the CERN Large Hadron Collider (LHC) operating at $\sqrt{S}=14 \mathrm{TeV}$ with projected data sets of $10-100 \mathrm{fb}^{-1}$ of integrated luminosity, SUGRA models predict the possibility of detection of directly produced slepton pairs [11,14]. This is likely possible in gauge-mediated models as well. SUGRA models predicting slepton masses of $100-400 \mathrm{GeV}$ ought to be detectable; this mass range also corresponds to the most favored range of slepton masses expected if the lightest neutralino makes up the bulk of dark matter in the universe [15].

Direct slepton pair event rates will be low at an upgraded Tevatron, while at the LHC, slepton reconstruction will be difficult. Theoretical improvements of the Born level analyses $[10,11]$ of slepton pair production are essential. In this paper, we present the leading quantum chromodynamics (QCD) corrections to the direct production of slepton pairs at hadron colliders. The calculation is described and formulae are given in Sec. II. Numerical results are shown for the Fermilab Tevatron and CERN LHC collider in Sec. III. The main result is that the slepton pair production cross sections are enhanced by $35-40 \%$ for the Fermilab Tevatron collider, and $25-35 \%$ for the CERN LHC collider relative to the leading order results. The theoretical uncertainties are addressed and it is found that the total cross section results should be accurate to $\pm 15 \%$. Finally, we comment upon the implications of these results for slepton search experiments. 


\section{ANALYTICAL RESULTS}

In this section we briefly describe the calculation of QCD corrections to the direct production of slepton pairs at hadron colliders and then give the corresponding cross section formulae. The results are very similar to those of the leading QCD corrections [16] to the SM Drell-Yan [17] process. The lowest order cross sections have been discussed in Refs. [18] and [11].

The colorless nature of the slepton pair final state allows one to factorize the production cross section into hadronic and SUSY pieces. The SUSY piece corresponds to the decay of a virtual SM gauge boson of mass $Q^{2}$ into a pair of slepton particles with masses $m_{1}$ and $m_{2}$. The decays are

$$
\begin{aligned}
& W^{*} \rightarrow \widetilde{\ell}_{L} \overline{\widetilde{\nu}}_{\ell L}, \\
& Z^{*}\left(\text { or } \gamma^{*}\right) \rightarrow \widetilde{\ell}_{L} \overline{\mathscr{Z}}_{L} \text {, } \\
& Z^{*}\left(\text { or } \gamma^{*}\right) \rightarrow \widetilde{\ell}_{R} \overline{\bar{\ell}}_{R} \text {, }
\end{aligned}
$$

and

$$
Z^{*} \rightarrow \widetilde{\nu}_{L} \overline{\tilde{\nu}}_{L}
$$

Using the gauge invariance of the hadronic piece and the slepton Feynman rules, from Ref. [19] for example, the two body final state phase space integrals in the SUSY piece may be performed analytically. The result is proportional to $\beta^{3}$ where

$$
\beta=\left[1-\frac{2\left(m_{1}^{2}+m_{2}^{2}\right)}{Q^{2}}+\frac{\left(m_{1}^{2}-m_{2}^{2}\right)^{2}}{Q^{4}}\right]^{1 / 2} .
$$

Having calculated the SUSY piece, the QCD corrections to the hadronic piece are standard and correspond to the production of a virtual SM gauge boson. Gluon-bremsstrahlung is added to the leading order processes, giving the subprocesses

$$
q \bar{q}^{\prime} \rightarrow W^{*} g
$$

and

$$
q \bar{q} \rightarrow Z^{*}\left(\text { or } \gamma^{*}\right) g
$$

Singularities corresponding to soft gluon emission cancel when the ultra-violet renormalized virtual diagram contributions are added. The remaining singularities are initial state collinear in origin and are mass factorized into scale dependent parton distribution functions. We use the modified minimal subtraction ( $\overline{\mathrm{MS}})$ scheme throughout.

At this order, in addition to the annihilation processes (2.3) there are also contributions from Compton scattering diagrams that may be obtained from those of Eq. (2.3) by crossing. There are no soft singularities for these processes, only initial state collinear singularities.

The total production cross section is given by a convolution of scale dependent parton distribution functions and hard scattering partonic subprocess cross sections:

$$
\begin{aligned}
\sigma= & \int_{\left(m_{1}+m_{2}\right)^{2}}^{S} d Q^{2} \int_{\frac{Q^{2}}{S}}^{1} d x_{A} \int_{\frac{Q^{2}}{S x_{A}}}^{1} d x_{B} \\
& \times\left\{\sum_{i j=q, \bar{q}} f_{i / A}\left(x_{A}, \mu_{f}\right) f_{j / B}\left(x_{B}, \mu_{f}\right) \frac{d \hat{\sigma}_{q q}}{d Q^{2}}\right. \\
& +\sum_{i=q_{,}, \bar{q}^{2}}\left[f_{i / A}\left(x_{A}, \mu_{f}\right) f_{g / B}\left(x_{B}, \mu_{f}\right)\right. \\
& \left.\left.+f_{g / A}\left(x_{A}, \mu_{f}\right) f_{i / B}\left(x_{B}, \mu_{f}\right)\right] \frac{d \hat{\sigma}_{q g}}{d Q^{2}}\right\},
\end{aligned}
$$

where $f_{i / A}\left(x, \mu_{f}\right)$ are the parton distribution functions for parton $i$ in hadron $A$ with momentum fraction $x$ evaluated at the factorization scale $\mu_{f}$. $S$ is the hadron-hadron center of mass energy squared which is related to $\hat{s}$, the parton-parton center of mass energy squared, via $\hat{s}=x_{A} x_{B} S$. Defining $z$ $=Q^{2} / \hat{s}$ the hard scattering partonic subprocess cross sections are given by

$$
\begin{aligned}
\frac{d \hat{\sigma}_{q q}}{d Q^{2}}= & \sigma_{0}\left\{\delta(1-z)+\frac{\alpha_{s}\left(\mu_{r}\right)}{2 \pi} \frac{4}{3}\left[4\left(1+z^{2}\right)\left(\frac{\ln (1-z)}{1-z}\right)+\right.\right. \\
& -2 \frac{\left(1+z^{2}\right)}{1-z} \ln z+\left(\frac{2 \pi^{2}}{3}-8\right) \delta(1-z) \\
& \left.\left.+\frac{3}{2} P_{q q}(z) \ln \frac{Q^{2}}{\mu_{f}^{2}}\right]\right\}
\end{aligned}
$$

and

$$
\begin{aligned}
\frac{d \hat{\sigma}_{q g}}{d Q^{2}}= & \sigma_{0} \frac{\alpha_{s}\left(\mu_{r}\right)}{2 \pi} \frac{1}{2}\left[\frac{3}{2}+z-\frac{3}{2} z^{2}+2 P_{q q}(z)\right. \\
& \left.\times\left(\ln \frac{(1-z)^{2}}{z}-1+\ln \frac{Q^{2}}{\mu_{f}^{2}}\right)\right]
\end{aligned}
$$

where $\sigma_{0}$ are given below, $\mu_{r}$ is the renormalization scale, and $P_{q q}$ and $P_{q g}$ are the splitting kernels [20] given by

$$
\begin{aligned}
& P_{q q}(z)=\frac{4}{3}\left[\frac{1+z^{2}}{(1-z)_{+}}+\frac{3}{2} \delta(1-z)\right] \\
& P_{q g}(z)=\frac{1}{2}\left[z^{2}+(1-z)^{2}\right] .
\end{aligned}
$$

For the $W$ exchange process,

$$
\sigma_{0}=\frac{g^{4}}{576 \pi} \frac{\beta^{3}}{2} z\left|D_{W}\left(Q^{2}\right)\right|^{2}
$$

with

$$
D_{X}\left(Q^{2}\right)=\frac{1}{Q^{2}-M_{X}^{2}+i \Gamma_{X} M_{X}} .
$$

For the $\gamma-Z$ exchange processes, 


$$
\sigma_{0}=\frac{g^{4}}{576 \pi} \frac{\beta^{3}}{2} z \frac{1}{4 \cos ^{4} \theta_{W}} \mathcal{F}_{\gamma Z}\left(Q^{2}\right)
$$

with

$$
\begin{aligned}
\mathcal{F}_{\gamma Z}\left(Q^{2}\right)= & \left|D_{Z}\left(Q^{2}\right)\right|^{2}\left(L_{q}^{2}+R_{q}^{2}\right) c_{\phi}^{2}+\frac{32 \cos ^{4} \theta_{W} e^{4} e_{\phi}^{2} e_{q}^{2}}{g^{4}} \frac{1}{Q^{4}} \\
& +2\left(L_{q}+R_{q}\right) c_{\phi} \frac{4 \cos ^{2} \theta_{W} e^{2} e_{\phi} e_{q}}{g^{2}} \\
& \times \frac{Q^{2}-M_{Z}^{2}}{Q^{2}}\left|D_{Z}\left(Q^{2}\right)\right|^{2} .
\end{aligned}
$$

The constants in $\mathcal{F}_{\gamma Z}$ are given by

$$
\begin{aligned}
& T_{3}^{f}= \pm 1 / 2 \quad(\text { weak isospin }), \\
& e_{f}=2 / 3,-1 / 3,-1,0 \quad \text { (electric charge), } \\
& L_{f}=2 T_{3}^{f}-2 e_{f} \sin ^{2} \theta_{W}, \\
& R_{f}=-2 e_{f} \sin ^{2} \theta_{W},
\end{aligned}
$$

and depending on whether one has a left- or right- SUSY partner,

$$
c_{\phi}=L_{f} \text { or } R_{f}
$$

for slepton $\widetilde{f}$.

\section{NUMERICAL RESULTS AND IMPLICATIONS FOR DETECTION}

Using the results described in the previous section a program was constructed to calculate slepton pair production cross sections according to Eq. (2.4). For input, standard values of the electroweak parameters were used [2] along with the CTEQ4M parton distribution functions [21]. A twoloop expression for $\alpha_{s}$ was used with fixed $n_{f}=5$. The value of $\Lambda_{Q C D}^{n_{f}=5}$ was taken from the parton distribution set.

The next-to-leading order (NLO) cross section results are presented in Fig. 1 for (a) $p \bar{p}$ collisions at $\sqrt{S}=2 \mathrm{TeV}$ and (b) $p p$ collisions at $\sqrt{S}=14 \mathrm{TeV}$ as a function of slepton mass. For $\tilde{\ell}_{L} \tilde{\nu}_{L}$ production, we have assumed $m \tilde{\ell}_{L}=m_{\tilde{\nu}_{L}}$. Then for both the Tevatron and the LHC, we see that $W^{*}$ $\rightarrow \widetilde{\ell}_{L} \tilde{\nu}_{L}$ is the dominant production mechanism, while $\widetilde{\ell}_{R} \overline{\mathscr{\ell}}_{R}$ is the smallest. The $\tilde{\ell}_{L} \overline{\mathscr{V}}_{L}$ and $\tilde{\nu}_{L} \overline{\tilde{\nu}}_{L}$ cross sections are intermediate between these two cases, and are of comparable magnitude to each other.

The results of Fig. 1 were produced by setting the renormalization scale $\mu_{r}$ equal to the factorization scale $\mu_{f}$; the square of the scale was taken to be the invariant mass of the slepton pair $Q^{2}$. To get some idea of the size of the uncalculated higher order terms we varied the scale from $Q / 2$ to $2 Q$. The results changed by $\pm 5 \%$ for the Tevatron and $\pm 2 \%$ for the LHC. The largest uncertainty comes from the parton distribution functions (PDFs). For a crude estimate we use a set that assumes a different functional form for the gluon and sea quark at the initial evolution scale, the Martin-Roberts-

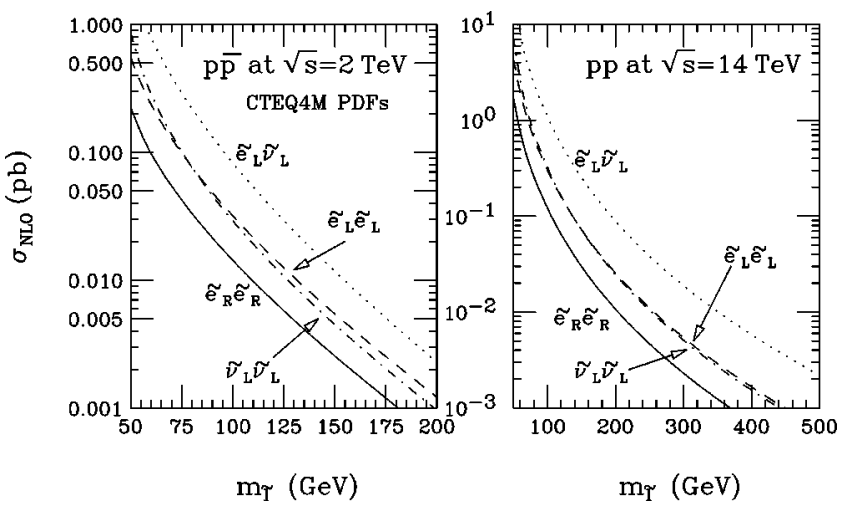

FIG. 1. The NLO total cross section for pair production of a single generation of sleptons: $\overline{\widetilde{e}}_{L} \widetilde{\nu}_{L}+\widetilde{e}_{L} \overline{\widetilde{\nu}}_{L}, \overline{\widetilde{e}_{L}} \widetilde{e}_{L}, \overline{\widetilde{e}}_{R} \widetilde{e}_{R}$ and $\widetilde{\nu}_{L} \widetilde{\nu}_{L}$, via the Drell-Yan mechanism versus slepton mass, for (a) $p \bar{p}$ collisions at $\sqrt{S}=2 \mathrm{TeV}$ and (b) $p p$ collisions at $\sqrt{S}=14 \mathrm{TeV}$. The slepton masses are assumed to be degenerate. We convolute with CTEQ4M parton distribution functions.

Stirling (MRS) set R1 [22]. This leads to an increase in cross section of approximately $10 \%$ for both Tevatron and LHC. We therefore cautiously estimate the theoretical uncertainty to be $\pm 15 \%$.

In Fig. 2, we plot the ratio of next-to-leading order cross sections to leading order cross sections, again versus slepton mass for the Tevatron and LHC colliders. The leading order results were computed using CTEQ4L parton distribution functions.

For the Tevatron collider, the various cross sections have an enhancement of $36-39 \%$ depending upon the slepton mass. The correction is nearly the same for all four slepton pair production reactions, as might be expected by the form of the correction given in Eqs. (2.5) and (2.6).

The most promising detection strategy for direct production of sleptons at the Tevatron in SUGRA-type models is via detection of acollinear dilepton pairs from, for instance, $p \bar{p} \rightarrow \widetilde{\ell}_{R} \overline{\mathscr{\ell}}_{R} \rightarrow \ell \bar{\ell}+\boldsymbol{E}_{T}$. Detailed simulations of this reaction have been performed in Ref. [11]. The major SM background to the clean dilepton signal comes from $W W \rightarrow \ell^{\prime}$ $+\boldsymbol{E}_{T}$. Reference [11] evaluated two case studies for slepton
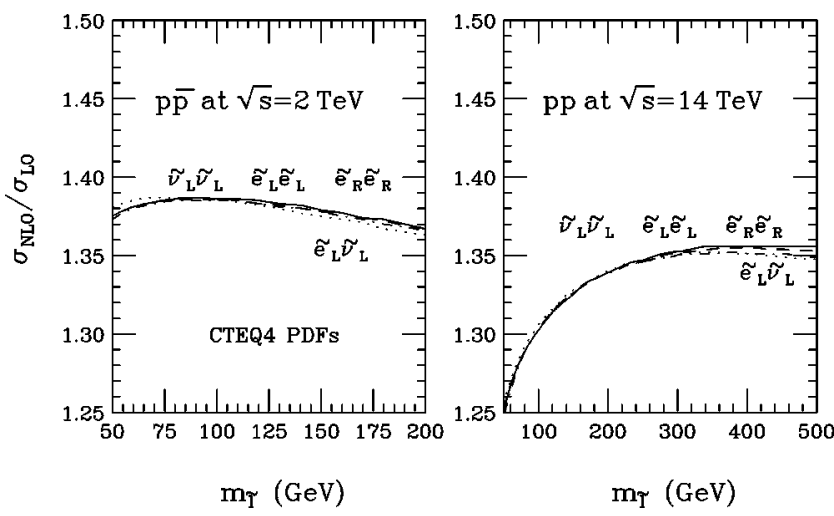

FIG. 2. The ratio of NLO to LO slepton pair production cross sections versus slepton mass, for (a) $p \bar{p}$ collisions at $\sqrt{S}=2 \mathrm{TeV}$ and (b) $p p$ collisions at $\sqrt{S}=14 \mathrm{TeV}$. The slepton masses are assumed to be degenerate. We convolute with CTEQ4M PDFs in the NLO case and CTEQ4L PDFs in the LO case. 
pair production. After cuts, case 1 , with $m_{\tau_{R}} \simeq 80 \mathrm{GeV}$, had $18 \mathrm{fb}$ of signal, while case 2 with $m_{\ell_{R}} \simeq 102 \mathrm{GeV}$ had $9 \mathrm{fb}$ of signal. The background from $W W$ at lowest order was evaluated at $36 \mathrm{fb}$. Thus, with $2 \mathrm{fb}^{-1}$ of integrated luminosity, neither signal case would be visible by itself at the $5 \sigma$ level. Incorporation of the NLO enhancement to both signal and background ( $K \simeq 1.3$ for $W W$ production at the Tevatron [23]) pushes case 1 above the $5 \sigma$ level, and into observability. In general, for the Tevatron, supersymmetric clean dilepton pair events can also come from chargino/neutralino pair production, and the net signal would likely come from a variety of different sparticle production reactions. The reach for SUGRA type SUSY models in the clean dilepton channel has been presented in Ref. [24].

For the case of the CERN LHC collider, Fig. 2(b) shows that the various slepton pair cross sections have an enhancement of $25-36 \%$ depending upon slepton mass. In Refs. $[11,14]$, it was found that a clean $\ell \vec{\ell}+\boldsymbol{E}_{T}$ signal could be seen above SM backgrounds for $100 \leqq m_{\tau_{R}} \subseteq 400 \mathrm{GeV}$. With hard enough cuts, the signal was shown to come almost exclusively from slepton pair production rather than from other SUSY particle production processes. However, direct reconstruction of parent slepton masses would be extremely difficult. Possibly the best option for a slepton mass measurement at the LHC might be from matching the total signal rate to expected rates from simulations of slepton production and decay for different mass sleptons. In this case, the most precise estimate of the slepton production cross sections would be needed.

\section{CONCLUSIONS}

The total cross sections for various slepton pair production reactions $\tilde{\ell}_{L} \tilde{\nu}_{L}, \tilde{\ell}_{L} \overline{\mathscr{\ell}}_{L}, \widetilde{\ell}_{R} \overline{\bar{\ell}}_{R}$ and $\tilde{\nu}_{L} \overline{\tilde{\nu}}_{L}$ were computed in next-to-leading order QCD. For $p \bar{p}$ collisions at $\sqrt{S}=2 \mathrm{TeV}$, leading order cross sections were enhanced by typically $35 \%$ to $40 \%$. For $p p$ collisions at $\sqrt{S}=14 \mathrm{TeV}$, the enhancement ranges from $25 \%$ to $35 \%$ depending on the mass of the sleptons. The theoretical uncertainty resulting from variations in the scale and parton distributions was found to be approximately $\pm 15 \%$. The NLO enhancements of the cross sections at Tevatron energies push some predictions for signals at the MI above the $5 \sigma$ level. At the LHC, where a slepton mass measurement may be made indirectly on the basis of event rates rather than by reconstruction, the NLO predictions for slepton pair production rates would be essential.

\section{ACKNOWLEDGMENTS}

We thank X. Tata for comments on the manuscript. This research was supported in part by the U.S. Department of Energy under contract number DE-FG05-87ER40319 and National Science Foundation grant PHY-9507688.
[1] X. Tata, in Proceedings of the Theoretical Advanced Study Institute in Elementary Particle Physics (TASI '95), Boulder, Colorado, 1995, edited by D. E. Soper (World Scientific, Singapore, 1996), p. 163; M. Drees, Report No. APCTP-05, hep-ph/9611409; S. Martin, hep-ph/9709356; for a phenomenological review, see H. Baer et al., in Electroweak Symmetry Breaking and New Physics at the TeV Scale, edited by $\mathrm{T}$. Barklow, S. Dawson, H. Haber, and J. Seigrist (World Scientific, Singapore, 1996), p. 216.

[2] See Particle Data Group, R.M. Barnett et al., Phys. Rev. D 54, 1 (1996).

[3] CDF Collaboration, F. Abe et al., Phys. Rev. Lett. 76, 2006 (1996).

[4] D0 Collaboration, S. Abachi et al., Phys. Rev. Lett. 75, 618 (1995).

[5] W. Beenakker, R. Hopker, M. Spira, and P. M. Zerwas, Phys. Rev. Lett. 74, 2905 (1995); Z. Phys. C 69, 163 (1995); Nucl. Phys. B492, 51 (1997).

[6] ALEPH Collaboration, R. Barate et al., CERN-PPE-97-128, hep-ex/9710012; Delphi Collaboration, P. Abreu et al., Eur. Phys. J. C1 1 (1998); L3 Collaboration, M. Acciarri et al., CERN-PPE-97-130; OPAL Collaboration K. Ackerstaff et al., CERN-PPE-97-083, hep-ex/9708018.

[7] ALEPH Collaboration, R. Barate et al., Phys. Lett. B 407, 377 (1997); Delphi Collaboration, P. Abreu et al., ibid. 387, 651 (1996); L3 Collaboration, M. Acciarri et al., see Ref. [6]; OPAL Collaboration, K. Ackerstaff et al., Phys. Lett. B 396, 301 (1997).

[8] L3 Collaboration, O. Adriani et al., Phys. Rep. 236, 1 (1993).
[9] CDF Collaboration, F. Abe et al., Phys. Rev. Lett. 76, 4307 (1996); D0 Collaboration, B. Abbott et al., ibid. 80, 1591 (1998).

[10] F. del Aguila and Ll. Ametller, Phys. Lett. B 261, 326 (1991).

[11] H. Baer, C. H. Chen, F. Paige, and X. Tata, Phys. Rev. D 49, 3283 (1994).

[12] M. Dine, A. Nelson, Y. Nir, and Y. Shirman, Phys. Rev. D 53, 2658 (1996).

[13] S. Dimopoulos, S. Thomas, and J. Wells, Phys. Rev. D 54, 3283 (1996); S. Ambrosanio, G. Kane, G. Kribs, S. Martin, and S. Mrenna, ibid. 54, 5395 (1996); H. Baer, M. Brhlik, C. H. Chen, and X. Tata, ibid. 55, 4463 (1997).

[14] D. Denegri, W. Majerotto, and L. Rurua, CMS-NOTE-1997094, hep-ph/9711357.

[15] H. Baer and M. Brhlik, Phys. Rev. D 53, 597 (1996).

[16] G. Altarelli, R. K. Ellis, and G. Martinelli, Nucl. Phys. B157, 461 (1979).

[17] S. Drell and T. Yan, Phys. Rev. Lett. 25, 316 (1970).

[18] S. Dawson, E. Eichten, and C. Quigg, Phys. Rev. D 31, 1581 (1985).

[19] H. E. Haber and G. L. Kane, Phys. Rep. 117, 75 (1985).

[20] G. Altarelli and G. Parisi, Nucl. Phys. B126, 298 (1977).

[21] CTEQ Collaboration, H. L. Lai et al., Phys. Rev. D 55, 1280 (1997).

[22] A. D. Martin, R. G. Roberts, and W. J. Stirling, Phys. Lett. B 387, 419 (1996).

[23] J. Ohnemus, Phys. Rev. D 44, 1403 (1991).

[24] H. Baer, C. H. Chen, C. Kao, and X. Tata, Phys. Rev. D 52, 1565 (1995); H. Baer, C. H. Chen, F. Paige, and X. Tata, ibid. 54, 5866 (1996). 